\title{
Dose-effect relationship of CpG oligodeoxyribonucleotide 1826 in murine Lewis lung cancer treated with irradiation
}

This article was published in the following Dove Press journal:

OncoTargets and Therapy

21 May 2013

Number of times this article has been viewed

\author{
Xibing Zhuang' \\ Tiankui Qiao' \\ Sujuan Yuan' \\ Wei Chen' \\ Lin Zha ${ }^{2}$ \\ Li Yan'
}

'Department of Oncology, Jinshan Hospital, Medical Center of Fudan University, Shanghai, People's Republic of China; ${ }^{2}$ Department of Oncology, Tongling People's Hospital, Tongling, Anhui, People's Republic of China
Correspondence: Tiankui Qiao Department of Oncology, Jinshan Hospital, Medical Center of Fudan University, Shanghai 201508, People's Republic of China

Email qiaotk@yahoo.com.cn
Background: Cytosine-phosphate-guanine ( $\mathrm{CpG}$ ) oligodeoxyribonucleotides (ODNs), which induce signaling via Toll-like receptor 9 , have recently been suggested to enhance sensitivity to traditional therapies, including chemotherapy, in certain cancer cell lines. This study aimed to define the dose-effect relationship for CpG ODN 1826 in increasing radiosensitivity and its impact on immune function in a mouse model of Lewis lung cancer.

Methods: The tumor-bearing mouse model was induced by injecting Lewis lung cancer cells into the right anterior leg subcutaneously. Sixty-four C57BL/6 J mice were evenly randomized into eight groups, comprising: a control group; an irradiation group; a CpG ODN 0.15 group; a CpG ODN 0.3 group; a CpG ODN 0.45 group; a CpG 0.15 + irradiation group; a CpG 0.3 + irradiation group; and a CpG 0.45 + irradiation group. Tumor growth, serum tumor necrosis factor-alpha and interleukin-12 concentrations, spleen and thymus exponents, and effect of $\mathrm{CpG}$ on the secondary immune response were measured, and apoptosis of tumor cells was investigated using TdT-mediated dUTP nick end labeling (TUNEL) after treatment.

Results: Tumor volumes in the treated groups were smaller than in the control group, with those of the CpG 0.45 + irradiation group being the smallest. TUNEL showed that the apoptosis rate in all the active treatment groups was higher than in the control group. CpG ODN apoptosis rate, serum tumor necrosis factor-alpha and interleukin-12 levels, and the spleen and thymus exponent showed greater improvement in the groups receiving combination therapy of CpG ODN and irradiation than the control group or the group receiving irradiation alone. With the increasing concentration of CpG ODN 1826, its effect became more and more significant, meanwhile, inoculation of Lewis lung cancer cells failed in those CpG ODN-cured mice.

Conclusion: $\mathrm{CpG}$ ODNs dramatically increased the radiosensitivity of Lewis lung cancer and enhanced immune function in mice in a dose-related manner.

Keywords: apoptosis, Lewis lung cancer, $\mathrm{CpG}$ oligodeoxyribonucleotides, secondary immune response

\section{Introduction}

Lung cancer is a disease with one of the highest morbidity and mortality rates worldwide, and long-term survival remains poor. The majority of lung cancers are diagnosed at an advanced stage, when the opportunity for surgery has been missed. Radiotherapy is one of the most important therapeutic strategies in these patients, but its efficacy is not satisfactory, not only because of the emergence of resistance, but also because of reduced immune function during treatment.

Nonmethylated cytosine-phosphate-guanine $(\mathrm{CpG})$ dinucleotides, present in bacterial DNA and recognized via Toll-like receptor 9 on plasmacytoid dendritic cells and B cells, are known to induce both innate and adaptive immunity, ${ }^{1}$ with the 
production of cytokines, such as tumor necrosis factor-alpha (TNF- $\alpha$ ), interleukin (IL)-12, and interferon gamma, needed for development of antitumor immunity. ${ }^{2-4}$ Recent studies show that $\mathrm{CpG}$ oligodeoxyribonucleotides (ODNs) can directly induce apoptosis in cancer cells, alter the cell cycle, and increase the sensitivity of chemotherapy and radiotherapy. ${ }^{5-9}$ However, whether it has a dose-effect relationship when combined with radiotherapy has not been well investigated. On the basis of current studies, we used different doses of CpG ODN 1826 combined with fractionated irradiation (IR) to treat mice with Lewis lung cancer cell implants in order to investigate dose-relevant interactions between $\mathrm{CpG}$ ODNs and radiotherapy.

\section{Materials and methods Main instruments and reagents}

The Lewis lung cancer cells used in this study were kindly supplied by the Life Science Institute of Academy in Shanghai and cultured in RPMI-1640 medium (Biowest Therapeutics Inc, Vancouver, Canada) supplemented with $10 \%$ fetal bovine serum and $1 \%$ penicillin-streptomycin. The cultures were maintained at $37.8^{\circ} \mathrm{C}$ in a humidified atmosphere containing $5 \% \mathrm{CO}_{2}$. The phosphorothioate-modified CpG ODNs were provided by Shanghai Sangon Biological Engineering Technology and Service Limited Company (Shanghai, People's Republic of China). The sequence of CpG ODN 1826 was 5'-TCCATGACGTTCCTGACGTT-3'. ODNs were diluted with phosphate-buffered saline to a concentration of $1 \mathrm{~g} / \mathrm{L}$ and maintained at $-20^{\circ} \mathrm{C}$. Serum levels of TNF- $\alpha$ and IL-12 were measured using a standard Quantikine enzyme-linked immunosorbent assay kit from R\&D Systems (Minneapolis, MN). A TdT-mediated dUTP nick end labeling (TUNEL) kit purchased from Beijing Tianyin Biochemical Technology Limited Company (Beijing, People's Republic of China) was used to detect tumor cell apoptosis. A linear accelerator was used (Precise 5839, Elekta, Stockholm, Sweden).

\section{Tumor-bearing mouse model}

Female C57BL/6 J mice were provided by the Shanghai Experimental Animal Center and maintained in a specific pathogen-free grade animal room until 6-8 weeks of age and weighing $18-22 \mathrm{~g}$. They were housed at 6-7 per cage. The study was approved by the ethics committee of Jinshan Hospital, Shanghai, People's Republic of China. Approximately $4 \times 10^{6}$ Lewis lung cancer cells were injected subcutaneously into the right anterior leg of each mouse when the cells were in the exponential growth phase. A tumor bulb could be seen on the right anterior leg seven days after the tumor cells were injected. In total, there were 64 tumor-bearing mice in this experiment.

\section{Experimental methods}

The experiment was started when the tumors were $6-8 \mathrm{~mm}$ in diameter. The mice were evenly randomized into eight groups. There was no treatment in the control group. Injections of $\mathrm{CpG}$ ODN 1826 were given intraperitoneally as a single dose of $0.05 \mathrm{mg}, 0.1 \mathrm{mg}$, or $0.15 \mathrm{mg}$ three times daily, on days 1, 2, and 9; the total dose of CpG ODN 1826 administered was $0.15 \mathrm{mg}, 0.3 \mathrm{mg}$, and $0.45 \mathrm{mg}$. On the second day of the experiment, the nonanesthetized mice were tied to small boards, and their tumors were centered in a $3 \mathrm{~cm}^{2}$ field. Radiation was delivered five times to the tumor-bearing leg using a linear $\mathrm{x}$-ray accelerator unit with a single dose of 2.5 Gy on days $2-6$, for a total dose of 12.5 Gy. In the mice receiving both radiation and $\mathrm{CpG}$ ODN 1826, radiation was given three hours after administration of CpG ODNs. On day 16 , tumor-bearing mice (except for three surviving mice that became tumor-free) were euthanized for investigation.

\section{Efficacy in transplanted tumors}

Starting on day 5 after inoculation, we weighed all tumorbearing mice in the eight treatment groups on alternate days, and recorded their mental status, eating habits, behavioral characteristics, and any deaths. The effects of different doses of CpG ODN 1826 and IR were well demonstrated by a delay in tumor growth and the sensitization enhancement ratio. The absolute tumor growth delay (ATGD) is defined as the time (days) for tumors in the treatment groups (IR $\pm \mathrm{CpG}$ ODN 1826) to grow from the original mean volume to four times that volume minus the time (days) taken for the tumors in the untreated control group to reach the same size. ${ }^{10}$ The sensitization enhancement ratio (SER) is defined as the value of ATGD in different dose of CpG ODN 1826 combined with IR groups divided by the ATGD in IR group. To obtain the tumor growth curve, we used a vernier caliper to measure the tumor diameters, and mean values were calculated. Tumor volume was calculated as:

$$
\text { Tumor volume }=1 / 2 a b^{2} \pi(a>b)
$$

where $a$ is the maximum diameter of the tumor and $b$ is the maximum diameter perpendicular to $a$. The tumor inhibitory rate was calculated as:

Tumor inhibitory rate $=1-($ Mean tumor weight of treated group/mean tumor weight of control group) $\times 100 \%{ }^{11}$ 


\section{Cell apoptosis}

A TUNEL kit was used to detect apoptosis in tumor cells, whereby apoptotic cell nuclei stain brown and nonapoptotic cells stain blue. Cell apoptosis was observed under a microscope by two pathologists working independently. The apoptosis rate was calculated as follows: (number of apoptosis cells/total number of cells $) \times 100 \%{ }^{12}$

\section{Impact on immune function}

On day 16, the serum was collected and all spleen, thymus, and tumor tissue was removed from the mice. A standard Quantikine enzyme-linked immunosorbent assay kit from R\&D Systems was used to measure serum TNF- $\alpha$ and IL-12 levels. Mean spleen and thymus exponents were calculated as the weight of the spleen divided by the weight of the mouse from which the tumor had been removed. We also observed growth of Lewis lung cancer cells reinoculated into the mice that had been cured.

\section{Statistical analysis}

Statistical analysis was done using the Statistical Package for Social Sciences version 17.0 (SPSS Inc, Chicago, IL, USA). Data are expressed as the mean \pm standard deviation.
The Student's $t$-test was used to test for differences between the groups. Differences resulting in $P<0.05$ were considered to be statistically significant.

\section{Results}

\section{Mouse model}

Sixty-four tumor-bearing mice were successfully created and treated with different doses of CpG ODN 1826, fractionated local tumor IR, or both CpG ODN 1826 and IR. Eight nontreated mice served as a control group, of which three died between days 13 and 15. The mental status, eating habits, and behavioral characteristics of the mice worsened in the control group. With the concentration of $\mathrm{CpG}$ ODNs increasing, the general survival condition was improved in the treated groups. One mouse in the $\mathrm{CpG} 0.3+$ IR group and two surviving mice in the $\mathrm{CpG} 0.45+$ IR group became tumor-free.

\section{Tumor growth}

CpG ODN 1826 has potent antitumor effects in vivo. ${ }^{13}$ Our study was intended to determine if there is a synergistic effect between CpG ODN 1826 and IR in terms of tumor growth. As shown in Figure 1, from day 3 onwards, the

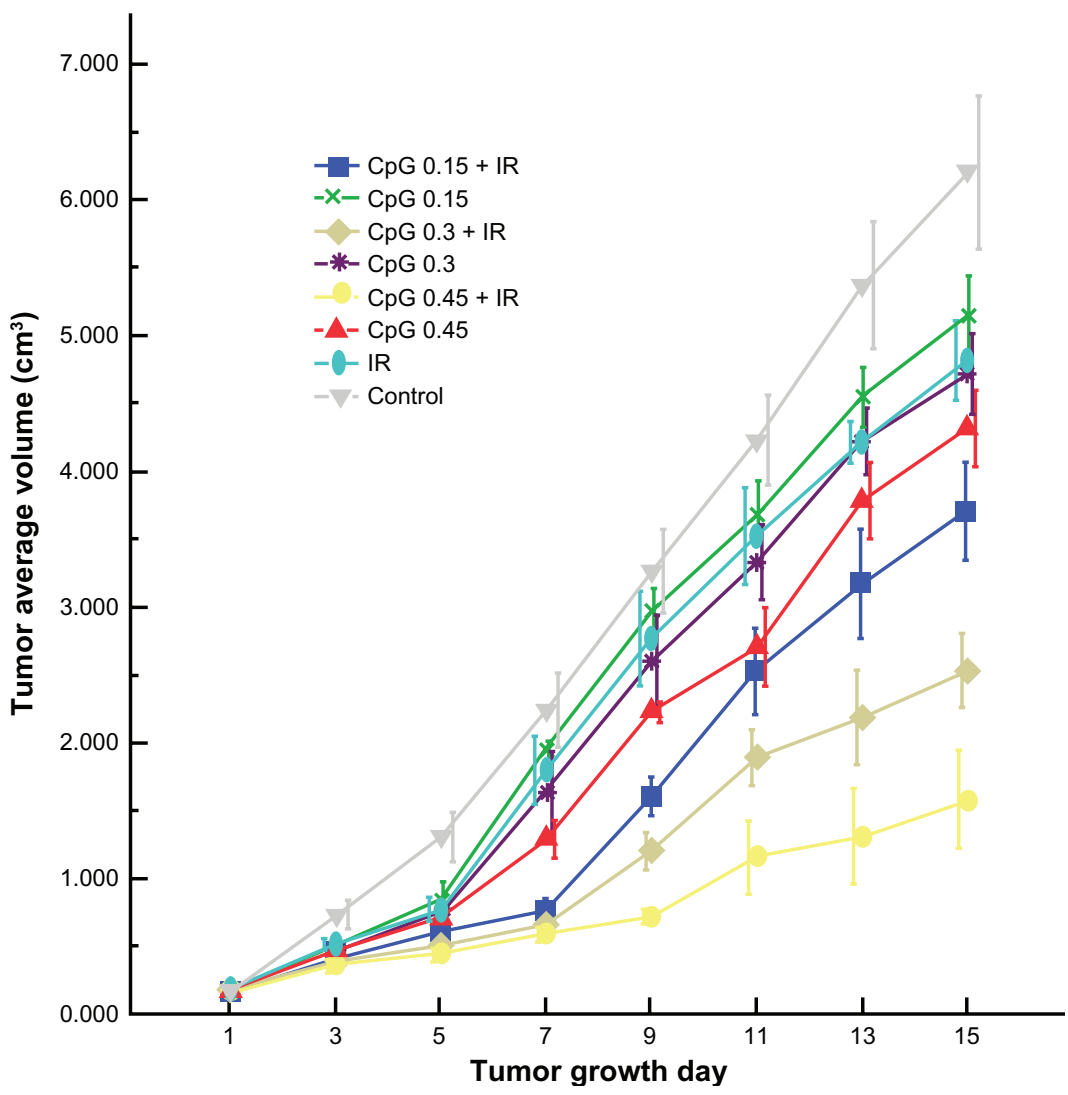

Figure I Tumor growth curve. Abbreviations: CpG, cytosine-phosphate-guanine; IR, irradiation. 
mean tumor volumes in the treatment groups were smaller than that in the control group, with the lowest tumor volume recorded in the $\mathrm{CpG} 0.45+\mathrm{IR}$ group. On the last day, the mean tumor volume was $6.21 \pm 0.45 \mathrm{~cm}^{3}$ in the control group, $4.82 \pm 0.34 \mathrm{~cm}^{3}$ in the irradiation group, $5.14 \pm 0.36 \mathrm{~cm}^{3}$ in the CpG ODN 0.15 group, $4.72 \pm 0.35 \mathrm{~cm}^{3}$ in the CpG ODN 0.3 group, $4.32 \pm 0.32 \mathrm{~cm}^{3}$ in the CpG ODN 0.45 group, $3.71 \pm 0.43 \mathrm{~cm}^{3}$ in the $\mathrm{CpG}$ ODN $0.15+\mathrm{IR}$ group, $2.53 \pm 0.29 \mathrm{~cm}^{3}$ in the $\mathrm{CpG}$ ODN $0.3+$ IR group, and $1.58 \pm 0.34 \mathrm{~cm}^{3}$ in the CpG ODN $0.45+$ IR group. There was no statistically significant difference in tumor volume between the groups before treatment while the tumor volume was remarkably decreased after treatment $(P<0.05)$. The therapeutic effect was most marked in the CpG ODN $0.45+$ IR group $(P<0.05)$. Table 1 shows that a total dose of 12.5 Gy given in 2.5 Gy fractions produced an ATGD of 2.0 days. CpG ODN 1826 alone delayed tumor growth for 1.8-2.3 days. In contrast, CpG ODN 1826 or IR was very effective, and the ATGD for these tumors was 4.4-6.0 days, with a sensitization enhancement ratio of 2.0, 2.7, and 3.0. This ATGD value was considerably higher than the sum of the tumor growth delay seen with each individual treatment, and this difference was statistically significant $(P<0.05)$. Moreover, ATGD and sensitization enhancement ratios increased with increasing concentrations of CpG ODN 1826.

\section{Cell apoptosis}

Apoptosis in Lewis lung cancer tumor cells in the control group and the groups treated with different doses of CpG ODN $1826 \pm$ IR was assessed by TUNEL. As shown in Table 2 and Figure 2, when mice were treated with different doses of $\mathrm{CpG}$ ODN 1826 or IR, the apoptosis rate was obviously higher than that in the nontreated mice $(P<0.05)$. Moreover, when

Table I Delay in tumor growth (days) and sensitization enhancement ratio

\begin{tabular}{lllll}
\hline Group & Mice (n) & Time & ATGD & SER \\
\hline Control & 5 & 1.8 & - & - \\
IR & 8 & 3.8 & 2.0 & - \\
CpG ODN 0.I5 & 8 & 3.6 & 1.8 & - \\
CpG ODN 0.3 & 8 & 3.8 & 2.0 & - \\
CpG ODN 0.45 & 8 & 4.5 & 2.3 & - \\
CpG ODN 0.I5 + IR & 8 & 6.2 & 4.4 & 2.2 \\
CpG ODN 0.3 + IR & 7 & 7.2 & 5.4 & 2.7 \\
CpG ODN 0.45 + IR & 6 & 7.8 & 6.0 & 3.0 \\
\hline
\end{tabular}

Note: Time taken (days) for tumors in the treatment groups (X-ray or CpG ODN 1826) to grow from their mean original volume to four times that volume.

Abbreviations: ATGD, absolute tumor growth delay; CPG, cytosine-guanine; IR, irradiation; n, number; ODN, oligodeoxyribonucleotide; SER, sensitization enhancement ratio.
Table 2 Apoptosis rate in tumor cells (mean \pm standard deviation)

\begin{tabular}{llll}
\hline Group & $\begin{array}{l}\text { Mice (n) } \\
\text { (start) }\end{array}$ & $\begin{array}{l}\text { Mice (n) } \\
\text { (end) }\end{array}$ & $\begin{array}{l}\text { Apoptosis } \\
\text { rate (\%) }\end{array}$ \\
\hline Control & 8 & 5 & $2.40 \pm 0.55$ \\
IR & 8 & 8 & $7.13 \pm 0.83$ \\
CPG ODN 0.15 & 8 & 8 & $5.50 \pm 0.76^{\mathrm{a}}$ \\
CPG ODN 0.3 & 8 & 8 & $11.63 \pm 1.06^{\mathrm{b}}$ \\
CPG ODN 0.45 & 8 & 8 & $19.13 \pm 0.83^{\mathrm{c}}$ \\
CPG ODN 0.15+ IR & 8 & 8 & $12.88 \pm 0.83^{\mathrm{a}, \mathrm{d}}$ \\
CPG ODN 0.3 + IR & 8 & 7 & $20.57 \pm 2.37^{\mathrm{a}, \mathrm{d}, \mathrm{e}}$ \\
CPG ODN 0.45 + IR & 8 & 6 & $28.17 \pm\left. 3.3\right|^{\mathrm{a}, \mathrm{d}, \mathrm{f}}$ \\
\hline
\end{tabular}

Notes: ${ }^{a} P<0.05$ versus control at $t=7.91,24.73,16.61$, and 17.05; ${ }^{\mathrm{b}} P<0.05$ versus CPG ODN 0.15 at $t=13.30 ;{ }^{c} p<0.05$ versus $C_{p}$ G ODN 0.3 at $t=15.72$; ${ }^{\mathrm{d} P}<0.05$ versus IR at $t=13.78,15.08$, and 17.47; ${ }^{\mathrm{e}} \mathrm{P}<0.05$ versus $C_{\mathrm{p}} \mathrm{O}$ ODN $0.15+\mathrm{IR}$ at $t=8.63 ;{ }^{\mathrm{f}} \mathrm{P}<0.05$ versus $\mathrm{CPG}$ ODN $0.3+\mathrm{IR}$ at $t=4.8 \mathrm{I}$.

Abbreviations: $C_{p}$, cytosine-guanine; IR, irradiation; n, number; ODN, oligodeoxyribonucleotides.

mice were treated with the two abovementioned therapies in combination, the apoptosis rate was significantly higher than when either treatment was administered alone $(P<0.05)$.

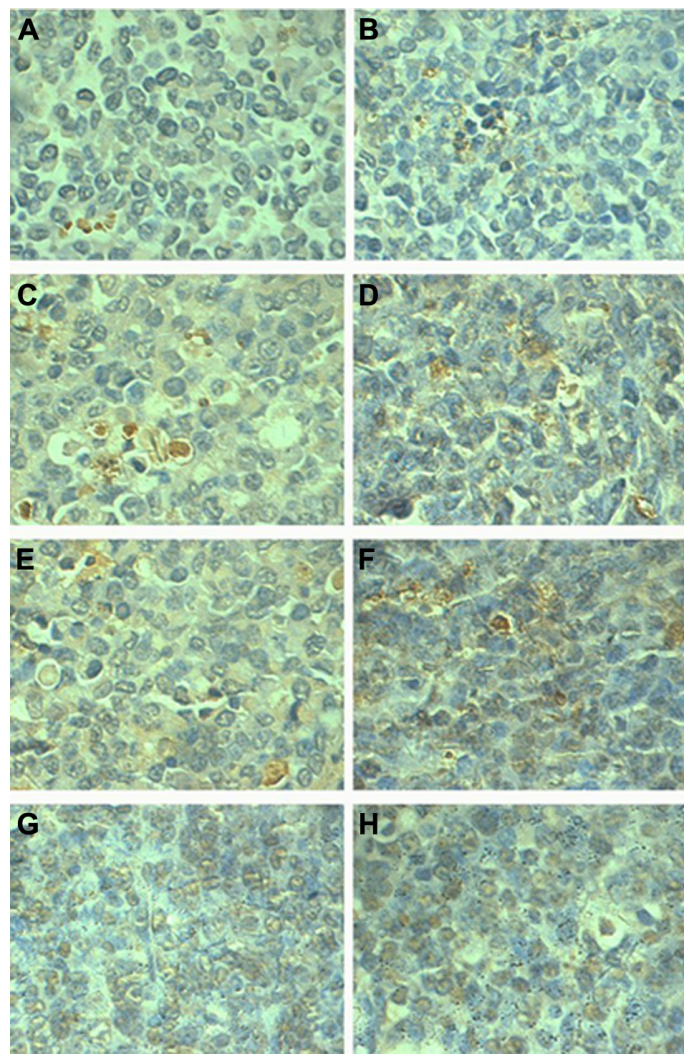

Figure 2 Apoptosis of cells in each experimental group $(\times 200)$. (A) Control group, (B) IR group, (C) CpG ODN 0.15 group, (D) CpG ODN 0.3 group, (E) CPG ODN 0.45 group, (F) CPG ODN $0.15+$ IR group, (G) CPG ODN $0.3+$ IR group, and (H) CPG ODN $0.45+$ IR group.

Abbreviations: CPG, cytosine-guanine; IR, irradiation; ODN, oligodeoxyribonucleotide. 
Further, efficacy increased with increasing doses of $\mathrm{CpG}$ ODN 1826.

\section{Impact on immune function}

As shown in Table 3, TNF- $\alpha$ and IL-12 levels were significantly lower in the group treated with IR alone than in the control group $(P<0.05)$, indicating that radiation might reduce immune function in mice. However, these indices were higher in the CpG ODN 1826 group than in the control group $(P<0.05)$, and there was no significant difference between the group receiving the low dose of $\mathrm{CpG}$ ODN 1826 and the control group. Meanwhile, compared with the IR group, TNF- $\alpha$ and IL-12 levels increased gradually when the mice were treated with increasing doses of $\mathrm{CpG}$ ODN $1826+\operatorname{IR}(P<0.05)$. A similar pattern of change in the spleen and thymus exponents was seen. Another research question was whether CpG ODN 1826 affected the secondary immune response, so Lewis lung cancer cells were reinoculated in the three tumor-free surviving mice. After ten days of observation, no transplanted tumors grew in these mice.

\section{Discussion}

Radiotherapy is the first-line treatment option for advanced cancer. However, immune function is severely compromised by radiotherapy, resulting in physical decline and limited therapeutic effect, and is a major limiting factor using this treatment modality. The innate immune system is geared towards providing a rapid response to foreign pathogens by pattern recognition receptors that distinguish prokaryotic from eukaryotic DNA. ${ }^{14}$ These receptors bind specifically to nonmethylated $\mathrm{CpG}$ dinucleotides, enabling bacteria and other pathogens to stimulate the innate immune system. Synthetic ODNs containing nonmethylated $\mathrm{CpG}$ motifs mimic bacterial DNA and can activate the immune response. They can also activate a multi- tude of immunocytes, generating a cascade of cytokines which trigger apoptosis and inhibit tumor growth. ${ }^{15}$ In light of these observations, we undertook this study to determine if $\mathrm{CpG}$ ODNs can reduce the immune suppression caused by radiation and thereby increase its therapeutic effect, and if so, whether there was a dose-response relationship for CpG ODNs.

The results of this study show that treatment using $\mathrm{CpG}$ ODN 1826 in mice bearing large established tumors markedly enhanced the tumoral response to fractionated IR. Enhancement of the treatment response was evident in the longer delay in tumor growth, an improved sensitization enhancement ratio, and a higher rate of apoptosis than that achieved by IR alone. $\mathrm{CpG}$ ODN 1826 or IR alone produced an ATGD of about 2.0 days while the different doses of CpG ODN 1826 + IR improved the ATGD to 4.4-6.0 days and the sensitization enhancement ratio to 2.0-3.0. In addition, the apoptosis rate in mice treated with CpG ODN 1826 or IR alone was significantly higher $(P<0.05)$. Moreover, when mice received both treatments, the apoptosis rate was significantly higher than that in mice which received either treatment alone. Further, with increasing doses of CpG ODN 1826, the antitumor effect became more significant. These results show that CpG ODN 1826 can induce apoptosis, and further, that it augments the effect of IR, with some degree of dose-response relationship.

What's more, a more powerful immune function, which was demonstrated by detecting the concentration of TNFand IL-12 in serum and the spleen and thymus exponent, than that from IR alone was observed in groups combined with CpG ODN 1826 and IR. There was no significant difference in effect between the group receiving the low dose of $\mathrm{CpG}$ ODN 1826 and the control group. With increasing doses of $\mathrm{CpG}$ ODN 1826 in the combination therapy groups, the immunoprotective effect became increasingly significant. In contrast, CpG ODN 1826 alone or combined with IR could increase

Table 3 Concentrations of TNF- $\alpha$, and spleen and thymus exponent (mean \pm standard deviation)

\begin{tabular}{|c|c|c|c|c|}
\hline Group & $\begin{array}{l}\text { Mice }(\mathrm{n}) \\
\text { (start/end) }\end{array}$ & $\begin{array}{l}\text { TNF- } \alpha \\
(\mu g / L)\end{array}$ & Spleen exponent $(\mathrm{mg} / \mathrm{g})$ & $\begin{array}{l}\text { Thymus exponent } \\
\text { (mg/g) }\end{array}$ \\
\hline Control & $8 / 5$ & $|26.84 \pm| 2.1 \mid$ & $8.88 \pm 1.60$ & $2.25 \pm 0.61$ \\
\hline IR & $8 / 8$ & $131.11 \pm 14.13$ & $9.66 \pm 2.17$ & $2.31 \pm 0.57$ \\
\hline CpG ODN 0.15 & $8 / 8$ & $138.97 \pm 9.27$ & $9.74 \pm 1.46$ & $2.58 \pm 0.42$ \\
\hline CpG ODN 0.3 & $8 / 8$ & $149.59 \pm 9.60^{\mathrm{a}, \mathrm{b}}$ & $12.49 \pm 1.56^{\mathrm{a}, \mathrm{b}}$ & $3.11 \pm 0.43^{\mathrm{a}, \mathrm{b}}$ \\
\hline CpG ODN 0.45 & $8 / 8$ & $|7| .8 \mid \pm 8.06^{\mathrm{a}, \mathrm{b}, \mathrm{c}}$ & $15.68 \pm 2.69^{\mathrm{a}, \mathrm{b}, \mathrm{c}}$ & $3.74 \pm\left. 0.4\right|^{\mathrm{a}, \mathrm{b}, \mathrm{c}}$ \\
\hline$C_{P G}$ ODN $0.15+I R$ & $8 / 8$ & $133.23 \pm 13.81$ & $9.89 \pm 2.23$ & $2.45 \pm 0.39$ \\
\hline CpG ODN 0.3 + IR & $8 / 8$ & $156.96 \pm 9.48^{\mathrm{d}, \mathrm{e}}$ & $|3.7| \pm 2.36^{\mathrm{d}, \mathrm{e}}$ & $3.06 \pm 0.62^{\mathrm{d}, \mathrm{e}}$ \\
\hline CpG ODN $0.45+$ IR & $8 / 8$ & $179.37 \pm 11.50^{\mathrm{d}, \mathrm{e}, \mathrm{f}}$ & $16.36 \pm 2.26^{\mathrm{d}, \mathrm{e}, \mathrm{f}}$ & $3.90 \pm 0.68^{\mathrm{d}, \mathrm{e}, \mathrm{f}}$ \\
\hline
\end{tabular}

Notes: ap $<0.05$ versus control at $t=3.770,8.104,4.026,5.062,3.008$, and 5.326; ${ }^{\mathrm{b} P}<0.05$ versus CPG ODN 0.15 at $t=2.249,7.557,3.644,5.482,2.496$, and 5.579 ;

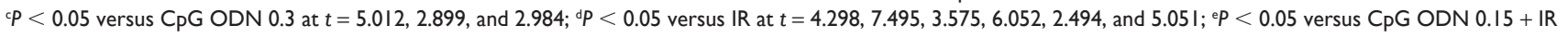
at $t=4.008,7.263,3.325,5.762,2.344$, and 5.222; $\mathrm{f} P<0.05$ versus $\mathrm{CpG}$ ODN $0.3+\operatorname{IR}$ at $t=4.255,2.303$, and 2.572 .

Abbreviations: $C_{P G}$, cytosine-guanine; IR, irradiation; ODN, oligodeoxyribonucleotides; TNF- $\alpha$, tumor necrosis factor-alpha. 
serum TNF- $\alpha$ and IL-12 levels and increased the spleen and thymus exponents, while IR alone decreased these parameters and immune activation, indicating a dose-dependent relationship. Moreover, in view of the failed reinoculation in tumor-free surviving mice, we hypothesize that CpG ODNs trigger production of cytokines like IL-12 and IL-18 in the body to activate memory lymphocytes, which is similar to the conclusion drawn by Kawarada et al. ${ }^{16}$ Our results show that CpG ODNs, a powerful inducer of antitumor immunity, can protect against radiotherapy-induced immune dysfunction and induce a memory immune response against tumor antigens.

Existing investigations have shown that CpG ODNs have not only the full range of stimulation to body's immune system but also the antitumor effects, which provided a spacious perspective for people in preventing and curing malignant tumors. However, these studies have only investigated the effects of CpG ODN 1826 in mice. Further research will be required to clarify the mechanism involved, the dose of $\mathrm{CpG}$ ODNs required, and the reason for more destructive effects in the human body than in mice.

\section{Acknowledgment}

This research was supported by the Shanghai Science and Technology Innovation Project (No. 2010-3-16).

\section{Disclosure}

The authors report no conflicts of interests in this work.

\section{References}

1. Krieg AM. CpG motifs in bacterial DNA and their immune effects. Annu Rev Immunol. 2002;20:709-760.

2. Akira S, Takeda K, Kaisho T. Toll-like receptors: critical proteins linking innate and acquired immunity. Nat Immunol. 2001;2(8):675-680.

3. Heckelsmiller K, Rall K, Beck S, et al. Peritumoral CpG DNA elicits a coordinated response of $\mathrm{CD} 8 \mathrm{~T}$ cells and innate effectors to cure established tumors in a murine colon carcinoma model. J Immunol. 2002; 169(7):3892-3899.
4. Mahfouz M, Hashimoto W, Das Gupta TK, Chakrabarty AM. Bacterial proteins and CpG-rich extrachromosomal DNA in potential cancer therapy. Plasmid. 2007;57(1):4-17.

5. Wang H, Rayburn ER, Wang W, Kandimalla ER, Agrawal S, Zhang R. Chemotherapy and chemosensitization of non-small cell lung cancer with a novel immunomodulatory oligonucleotide targeting Toll-like receptor 9. Mol Cancer Ther. 2006;5(6):1585-1592.

6. El Andaloussi A, Sonabend AM, Han Y, Lesniak MS. Stimulation of TLR9 with CpG ODN enhances apoptosis of glioma and prolongs the survival of mice with experimental brain tumors. Glia. 2006;54(6):526-535.

7. Petrangolini G, Tortoreto M, Perego P, et al. Combination of metronomic gimatecan and $\mathrm{CpG}$-oligodeoxynucleotides against an orthotopic pancreatic cancer xenograft. Cancer Biol Ther. 2008;7(4): 596-601.

8. Yuan S, Qiao T, Chen W. CpG oligodeoxynucleotide 1826 enhances the Lewis lung cancer response to radiotherapy in murine tumor. Cancer Biother Radiopharm. 2011;26(2):203-208.

9. Shen W, Waldschmidt M, Zhao X, Ratliff T, Krieg AM. Antitumor mechanisms of oligodeoxynucleotides with $\mathrm{CpG}$ and polyG motifs in murine prostate cancer cells: decrease of NF-kappaB and AP-1 binding activities and induction of apoptosis. Antisense Nucleic Acid Drug Dev. 2002;12(3): 155-164.

10. Mason KA, Neal R, Hunter N, Ariga H, Ang K, Milas L. CpG oligodeoxynucleotides are potent enhancers of radio- and chemoresponses of murine tumors. Radiother Oncol. 2006;80(2):192-198.

11. Zhao N, Wang L, Mou HY, et al. Synergism and attenuation effects of taurine on cyclophosphamide. Ai Zheng. 2009;28(3):244-248. Chinese.

12. Zhou HB, Chen JM, Cai JT, Du Q, Wu CN. Anticancer activity of genistein on implanted tumor of human SG7901 cells in nude mice. World J Gastroenterol. 2008;14(4):627-631.

13. Krieg AM. Development of TLR9 agonists for cancer therapy. J Clin Invest. 2007;117(5):1184-1194.

14. Medzhitov R, Janeway C Jr. Immune recognition: mechanisms and pathways. Immunol Rev. 2000;173:89-97.

15. Takeshita S, Takeshita F, Haddad DE, Ishii KJ, Klinman DM. $\mathrm{CpG}$ oligodeoxynucleotides induce murine macrophages to upregulate chemokine mRNA expression. Cell Immunol. 2000;206(2): 101-106.

16. Kawarada Y, Ganss R, Garbi N, Sacher T, Arnold B, Hämmerling GJ. NK- and CD8(+) T cell-mediated eradication of established tumors by peritumoral injection of $\mathrm{CpG}$-containing oligodeoxynucleotides. J Immunol. 2001;167(9):5247-5253.
OncoTargets and Therapy

\section{Publish your work in this journal}

OncoTargets and Therapy is an international, peer-reviewed, open access journal focusing on the pathological basis of all cancers, potential targets for therapy and treatment protocols employed to improve the management of cancer patients. The journal also focuses on the impact of management programs and new therapeutic agents and protocols on

Submit your manuscript here: http://www.dovepress.com/oncotargets-and-therapy-journal

\section{Dovepress}

patient perspectives such as quality of life, adherence and satisfaction. The manuscript management system is completely online and includes a very quick and fair peer-review system, which is all easy to use. Visit http://www.dovepress.com/testimonials.php to read real quotes from published authors. 